\title{
Zero-Mode Waveguide Detection of Flow-Driven DNA Translocation through Nanopores
}

\author{
Thomas Auger, ${ }^{1}$ Jérôme Mathé, ${ }^{2}$ Virgile Viasnoff, ${ }^{3}$ Gaëlle Charron, ${ }^{1}$ Jean-Marc Di Meglio, ${ }^{1}$ \\ Loïc Auvray, ${ }^{1}$ and Fabien Montel ${ }^{1}$ \\ ${ }^{1}$ Matiére et Systèmes Complexes, Université Paris Diderot \& CNRS (UMR 7057), 75205 Paris Cedex 13, France \\ ${ }^{2}$ Laboratoire d'Analyse et de Modélisation pour la Biologie et l'Environnement, \\ Université Évry-Val d'Essonne \& CNRS (UMR 8587), 91025 Évry Cedex, France \\ ${ }^{3}$ Mechanobiology Institute, National University of Singapore \& CNRS (UMI 3639), 5A Engineering Drive 1, \\ Singapore 117411, Singapore \\ (Received 24 February 2014; revised manuscript received 12 April 2014; published 9 July 2014)
}

\begin{abstract}
We directly measure the flow-driven injection of DNA through nanopores at the level of single molecule and single pore using a modified zero-mode waveguide method. We observe a flow threshold independent of the pore radius, the DNA concentration, and length. We demonstrate that the flow injection of DNA in nanopores is controlled by an energy barrier as proposed in the de Gennes-Brochard suction model. Finally, we show that the height of the energy barrier is modulated by functionalizing the nanopores.
\end{abstract}

DOI: 10.1103/PhysRevLett.113.028302

PACS numbers: 82.35.Lr, 87.15.hj, 87.80.Nj, 87.85.Qr

Flow injection of polymers into nanopores is a critical step of many industrial processes including filtration and oil recovery. It might also play an important role in future nanofluidic devices [1]. The most challenging regime where the polymer chains are larger than the pores was analyzed a long time ago by S. Daoudi, F. Brochard, and P.-G. de Gennes, using different approaches. In the "affine deformation model" [2,3] a flexible polymer coil is squeezed laterally by the flow converging at the entrance of a pore: the chain enters a pore when its transverse size becomes smaller than the pore diameter. This occurs when the flow rate $J$ is larger than a threshold $J_{c} \propto k_{B} T / \eta$, where $k_{B} T$ is the thermal energy and $\eta$ is the viscosity of the solvent. $J_{c}$ is surprisingly independent of pore size and polymer mass. Below $J_{c}$, the preceding description is not valid: one expects an increase in chain concentration at the pore entrance [3]. A calculation of the steady-state concentration profile in one-dimensional geometry is given in Ref. [4] and is easily generalized to the tridimensional case [5]. In the "suction model" [6], the chain is pulled in the pore by the friction force exerted by the flow on the monomers that have entered the pore. The chain enters the pore when the hydrodynamic drag forces overcome the entropic forces of confinement. This occurs above a threshold of flow rate that is identical to the one of the affine deformation model. Below the threshold, an energy barrier separates the state where the chain stays outside of the state where it fully invades the pore. Close to the threshold, the height of the barrier is of order $k_{B} T$ and the transition between the two states is smooth because of

Published by the American Physical Society under the terms of the Creative Commons Attribution 3.0 License. Further distribution of this work must maintain attribution to the author $(s)$ and the published article's title, journal citation, and DOI. thermal fluctuations. Each model focuses on different aspects of the injection, and a complete model is lacking; in particular, no theory predicts the exact value of the injection threshold beyond a scaling law derivation. Numerical studies confirm the universality of the threshold and the existence of a smooth crossover from retention to translocation [11].

Macroscopic flow experiments have evidenced the existence of a flow threshold by measuring the global rejection coefficient of filtration membranes [12-15]. But the nature of the transition from retention to translocation and the absolute value of the threshold are still debated. Indeed, in macroscopic experiments it is difficult to unravel the different contributions coming from the pores polydispersity, membrane polarization, and the injection barrier [14].

Here, we present results on the flow-driven translocation of long double-strand DNA molecules using a novel optical method which detects the translocation of single molecules through single pores in real time. We show that pressuredriven DNA transport in solid-state nanopores exhibits a flow threshold independent of pore radius, DNA length, and concentration. We also demonstrate that this threshold is smooth and is well described by an extension of the suction model. The exit time is measured as well and compared with the prediction of a simple hydrodynamical model. Taking advantage of the high sensitivity of our method, we also measure the effect of grafting short polymers at the pore exit on the height of the energy barrier.

Until now most of the experiments studying the translocation of molecules through nanopores have used an electrical driving force and an electrical detection [16]. Only a few experiments have used the potentiality of a pressure-driven transport $[17,18]$ or of an optical detection [19-21]. We developed a novel setup, described in Fig. 1, 

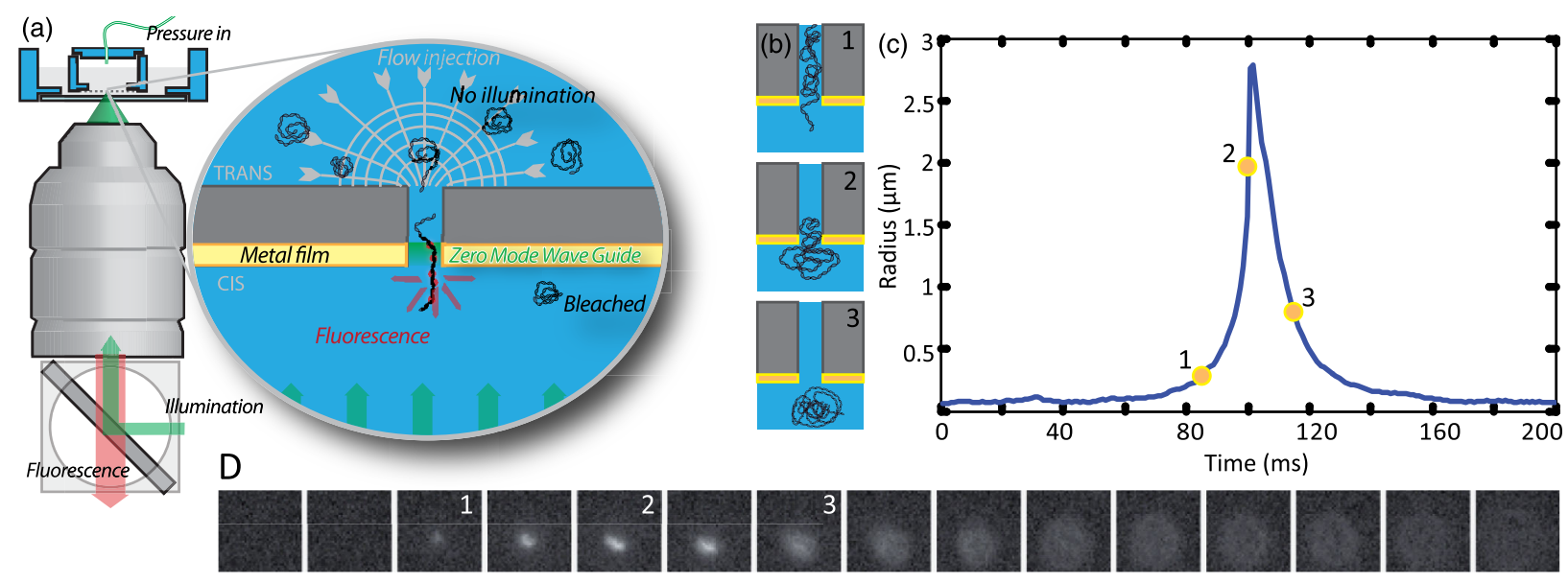

FIG. 1 (color online). (a) Zero mode waveguide setup for nanopore translocation. The trans chamber contains the YOYO-1 labeled DNA molecules. Upon pressure application, the DNA molecules are pushed through the pore and are illuminated as soon as they cross the evanescent field region at the end of the pore. Once they have left the pore, they are unfocused and bleached. (b) Schematics of the polymer exit. (c) Experimental evolution of the (thresholded) radius of the DNA fluorescent spot vs time. (D) Snapshots of a single DNA exit. Frame rate: $100 \mathrm{fps}$.

hat combines optical detection and pressure control in order to drive the translocation of polymers through solid-state nanopores. We used track-etched membranes (Whatman, with nominal pore diameters equal to $50,80,100$, or $200 \mathrm{~nm}$ and thicknesses equal to $6,6,6$, and $10 \mu \mathrm{m}$, respectively), coated with a thin layer of gold (Plassys MEB $550 \mathrm{~S}$ evaporator, thickness $50 \mathrm{~nm}$ and surface roughness $2.5 \mathrm{~nm}$ ). The membranes were illuminated from the (golden) cis-side by an extended laser beam. The gold layer induces a zero-mode waveguide illumination [22] at the end of the pores. We observed the transport of fluorescently labeled single molecules through the pores using an electron multiplying charge coupled device camera (Andor, iXon 897): molecules inside the pore were invisible until they reached the volume illuminated by the evanescent field. The molecules' fluorescence eventually disappeared because of bleaching and optical defocusing of molecules moving away from the membrane. The measurements were carried out at constant pressure in order to control the force applied on the molecules and to limit the influence of clogged pores on the flow rate. We used a pressure microcontroller (MFCS, Fluigent, Paris) with a pressure resolution better than $0.1 \mathrm{mbar}$.

A dilute solution of $\lambda$-phage DNA (1 pM, $48 \mathrm{kbp})$ in Tris and $\mathrm{KCl}$ buffer (both at $10 \mathrm{mM}, p H=7.4$ ) fluorescently labeled with Yoyo-1 (Life Tech) filled the trans-chamber where the pressure was applied as shown on Fig. 1(a). A few hundred translocation events were observed simultaneously with a time resolution of $10 \mathrm{~ms}$, which was sufficient to resolve each event as indicated in Figs. 1(b) and 1(d). The acquired movies were analyzed with a homemade MATLAB (Mathworks) program combining 3D median denoising and hysteresis threshold segmentation to determine the frequency $f_{\text {DNA }}$ of exiting events and the exit durations $t_{\text {exit }}$.
We analyzed the variation of the DNA translocation frequency per pore $f_{\mathrm{DNA}}$ with the pressure drop $P$. The data for pores of $50 \mathrm{~nm}$ diameter are plotted on Fig. 2(a). We observed a progressive increase of the translocation frequency revealing a smooth transition between total retention and unhindered transport.

In the framework of reaction rate theory, the translocation frequency is expressed as

$$
f_{\mathrm{DNA}}=K e^{-\Delta F / k_{B} T}
$$

assuming that DNA translocation is described as the travel of a particle through a free-energy landscape with a barrier of height $\Delta F . K\left(\mathrm{~s}^{-1}\right)$ is a rate of incidence on the barrier. When $K$ is dominated by the particle convection or by the presence of the barrier [23,24], $K \propto J$ with $J$ the solvant flux $\left(\mathrm{m}^{3} \mathrm{~s}^{-1}\right)$. In the suction model, the energy barrier is $\Delta F=k_{B} T\left(J_{c} / J\right)$ and the frequency of translocation $f_{\mathrm{DNA}}$ finally reads

$$
f_{\mathrm{DNA}}=k\left(P / P_{c}\right) e^{-P_{c} / P}
$$

with $k$ a proportionality factor $\left(\mathrm{s}^{-1}\right)$ and $P_{c}=R_{h} J_{c} ; R_{h}$ is the hydrodynamical resistance of the pore equal to $8 \eta L / \pi R^{4}$ with $L$ the length of a pore, $R$ the radius, and $\eta$ the solvent viscosity (Poiseuille law).

The pressure-frequency curves are well fitted by Eq. (2) (values of $k$ and $P_{c}$ in Table I). The frequencies normalized by $k$ are plotted as a function of the dimensionless expression $\left(P / P_{c}\right) \exp \left(-P_{c} / P\right)$ for pores of nominal diameters of 50, 80, 100, and $200 \mathrm{~nm}$ in Fig. 2(b). All data collapse on a master curve on more than 3 decades, in very good agreement with the suction model. We also checked that the critical pressure $P_{c}$ scales as $\left(L / R^{4}\right)$ as 

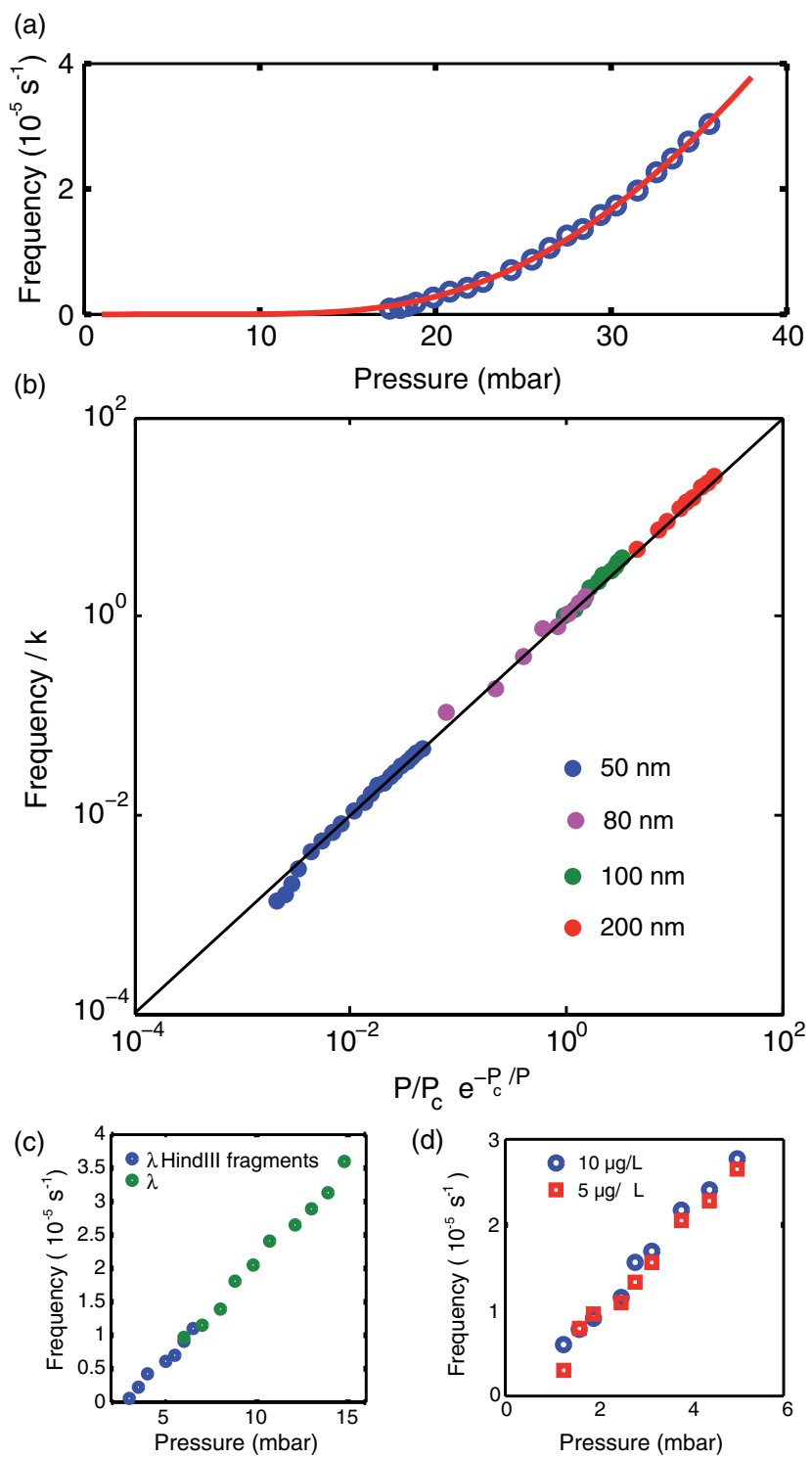

FIG. 2 (color online). (a) Translocation frequency vs pressure for $50 \mathrm{~nm}$ diameter pores. The continuous red curve is a fit by our model. (b) Normalized frequency of translocation per pore vs the dimensionless expression $P / P_{c} \exp \left(-P_{c} / P\right)$ for different pore diameters: $50 \mathrm{~nm}$ (blue), $80 \mathrm{~nm}$ (purple), $100 \mathrm{~nm}$ (green), $200 \mathrm{~nm}$ (red). (c) Effect of DNA length on the pressure-frequency behavior for $100 \mathrm{~nm}$ pores: translocation of DNA fragments obtained by restriction of $\lambda$ phage DNA through the enzyme HindIII normalized by the total number of molecules. (d) Effect of DNA concentration on pressure-frequency behavior for $200 \mathrm{~nm}$ pores. (The experimental errors are the standard error of the mean and are represented by the diameter of the data points, $N_{\text {events }}>800$ for each experimental series.)

expected from the Poiseuille law. We independently verified the applicability of the Poiseuille law for the macroscopic solvent flow in the nanoporous membranes using a microfluidic flow measurement system (Flowell, Fluigent). The critical flow $J_{c}$ is then remarkably constant over the range of pore diameters: we measured a critical flow rate of
TABLE I. Experimental results extracted from the pressurefrequency curves. $R_{\text {nom }}$ is the radius value given by the manufacturer, and $R_{\text {meas }}$ is the corresponding measured value using scanning electron microscopy. The critical pressure $P_{c}$ and the prefactor $k$ [Eq. (2)] uncertainties are extracted by bootstrapping on the fit through a Trust-Region algorithm. The threshold value $J_{c}$ is calculated using $J_{c}=P_{c} / R_{h}$ and assumes $\eta=0.001 \mathrm{~Pa} \cdot \mathrm{s}$.

\begin{tabular}{lcccl}
\hline \hline & & & & \\
$R_{\text {nom }}(\mathrm{nm})$ & $R_{\text {meas }}(\mathrm{nm})$ & $P_{\mathrm{c}}(\mathrm{mbar})$ & $k\left(10^{-6} \mathrm{~s}^{-1}\right)$ & $\left(10^{-2} k_{B} T / \eta\right)$ \\
\hline 25 & $21 \pm 0.25$ & $82 \pm 4$ & $713 \pm 92$ & $2.5 \pm 0.2$ \\
40 & $44 \pm 0.35$ & $3.8 \pm 4$ & $47.9 \pm 7.7$ & $2.4 \pm 0.2$ \\
50 & $45 \pm 0.35$ & $4 \pm 0.2$ & $14.4 \pm 12.6$ & $2.6 \pm 0.21$ \\
100 & $110 \pm 0.9$ & $0.2 \pm 0.01$ & $1.72 \pm 0.21$ & $2.8 \pm 0.22$ \\
\hline \hline
\end{tabular}

$0.026 \pm 0.002 k_{B} T / \eta$ (Table I). For flexible polymers, there is a dispersion of measured values in the literature ranging from 0.006 to 0.22 units of $k_{B} T / \eta$ [12-14]. We checked by electron microscopy of cut membranes that the pores of this study were actual cylinders. This study provides the first values of the critical flux for a semiflexible polymer such as DNA.

To test the independence of $J_{c}$ with respect to the DNA length, we performed experiments with molecules of different molecular weights obtained by cutting the original templates with restriction enzymes HindIII. The result was a DNA solution with six different lengths present in equal molar concentrations (from $2 \mathrm{kbp}$ to $23 \mathrm{kbp}$ ). The pressurefrequency curves before and after restriction are presented in Fig. 2(c) and are identical within experimental errors. We also varied the concentration of the DNA, and we observed that the critical pressure is independent of the concentration of the polymer as presented in Fig. 2(d). We conclude that we have experimental evidence that shows the independence of the critical flux $J_{c}$ on pore radius, concentration, and molecular weight.

Note that the proportionality factor $k$ introduced in Eq. (2) scales as $k \propto R_{\text {meas }}^{\alpha}$ with $\alpha=-3.5 \pm 0.3$ (Table I, second and fourth columns) and that the results of Fig. 2(d) also show that $k$ is independent of the DNA concentration. We have no straightforward arguments to explain these behaviors, which would clearly require a thorough theoretical analysis.

Our setup can provide the duration of the exit process of the DNA individual molecules. Indeed, the blurring at the end of the exit event can be used to precisely determine the beginning and the end of the translocation event as presented on Fig. 3(a). We measured this duration $t_{\text {exit }}$ as a function of the applied pressure $P$. The results for pore diameters of 50,100, and $200 \mathrm{~nm}$ are represented in Fig. 3(b): $t_{\text {exit }}$ scales linearly with the inverse of pressure. We can explain this behavior if we assume that the DNA molecules that have entered the pore are those that have crossed stochastically, pushed by the flow, the energy barrier. For zero-mode waveguides, the illumination depth 
(a)
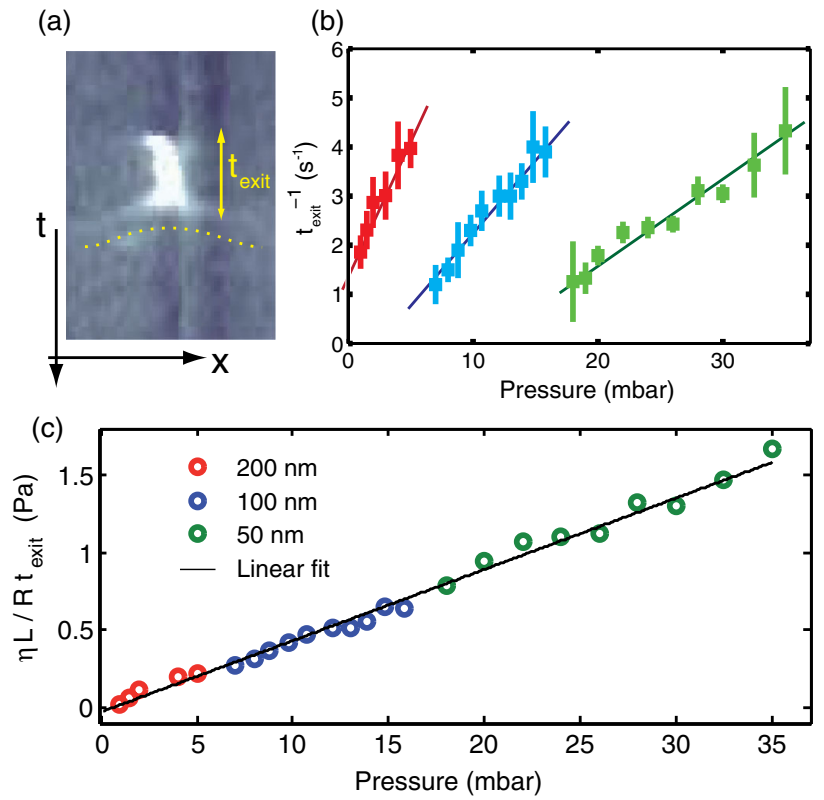

FIG. 3 (color online). (a) Determination of the event start and end. Evolution of the fluorescence intensity as a function of time and space. The start is characterized by a sharp increase of the intensity while the end is characterized by a blurring of the signal (underlined by the dotted line). (b) Evolution of the exit time $t_{\text {exit }}$ as a function of the applied pressure for 200 (blue), 100 (red), and 50 (green) nm pore diameter. Data extracted from three independent experiments. $N_{\text {events }}>800$ for each series. Straight lines are linear fits. (c) $\eta L / R t_{\text {exit }}$ as a function of $P$ for the same data series.

$\delta$ outside the pore is proportional to the radius of the pore [22]: $\delta \propto R$. The duration $t_{\text {exit }}$ of an exit event is then

$$
\left(t_{\text {exit }}\right)^{-1} \propto \frac{J}{\delta^{3}} \propto \frac{R^{4} P}{\delta^{3} \eta L} \propto \frac{R P}{\eta L} .
$$

We have plotted in Fig. 3(c) $\eta L / R t_{\text {exit }}$ as a function of $P$ for pore diameters of 50, 100, and $200 \mathrm{~nm}$. Again, all data collapse on a master curve in good agreement with this simple model and support the idea that our illumination is indeed in the zero-mode waveguide regime.

Finally we used our setup to test the effect of the pore entrance functionalization on the value of the energy barrier of translocation. We grafted short polyethylene glycol thiol molecules (mPEG thiol) using a protocol first proposed by Jovanovic-Talisman et al. [25]. The gold sputtered membranes were cleaned in nitric acid, then incubated with the PEG thiol molecules (356 g/mol, Polypure) during $1 \mathrm{~h}$ and rinsed in pure deionized water (Millipore). The modified membranes were then placed in the setup, and the pressurefrequency curves were measured and fitted with the suction model. We observed (Fig. 4) a clear shift of the curves towards higher pressures as compared to the bare membranes. More precisely, we observed a change of critical pressure from $82 \pm 4$ to $150 \pm 7$ mbar (ratio $1.83 \pm 0.17$ ).

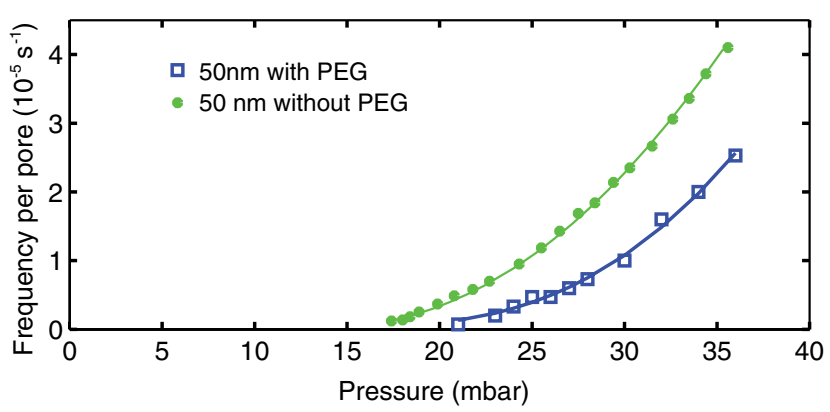

FIG. 4 (color online). Effect of mPEG thiol functionalization on the frequency of translocation for $50 \mathrm{~nm}$ pores. Data extracted from three independent experiments. The experimental errors are the standard error of the mean and are represented as the diameter of the data points. $N_{\text {events }}>800$ for each series. Full lines are fits of the data by our model.

This change can be related to the change of the effective radius of the pores. Indeed, the PEG thiol molecules have a total length of $3 \mathrm{~nm}$, which corresponds to a relative change of the effective pore radius of $14 \%$ and a relative increase of the critical pressure of 1.71 .

In summary, we have shown that a combination of nearfield optics and image analysis provides a powerful tool to study the translocation of individual biomolecules through solid-state nanopores. Using a pressure-controlled setup, we show that DNA molecules exhibit a flow threshold independent of the pore geometry and of their concentration and length. The pressure-frequency plots of translocation curves are readily fitted and explained by the suction model. We also demonstrate that the measured critical pressure can be used to investigate small modifications of the energy barrier through the reduction of the pore diameter. We believe that this method could be used to measure directly the interaction between translocated biomolecules and proteins grafted inside the pore. Our description neglects rigidity effects. We intend to study in the future the regime of strong confinement where the diameter of the pores is smaller than the persistence length of DNA.

We thank Françoise Brochard and Cyprien Gay for their fruitful comments and discussions. This work was supported in part by the Laboratoire d'Excellence "Who am I?" (PRES Sorbonne Paris Cité) and by the Agence Nationale de la Recherche under Grant No. ANR-12NANO-0012 Biograph'n.

[1] L. Bocquet and É. Charlaix, Chem. Soc. Rev. 39, 1073 (2010).

[2] S. Daoudi and F. Brochard, Macromolecules 11, 751 (1978).

[3] P.-G. de Gennes, Scaling Concepts in Polymer Physics (Cornell University Press, Ithaca, 1979).

[4] P.-G. de Gennes, C. R. Acad. Sci. Paris, Série IIb, 320, 501 (1995). 
[5] The monomer concentration profile $c(r)$ is self-similar and decays as $r^{-4 / 3}$.

[6] The suction model was first described as a private communication of P.-G. de Gennes in Ref. [7]. It is fully presented in Refs. [8-10].

[7] G. Guillot, Ph. D. Dissertation, University Paris Sud, 1986.

[8] K. Guevorkian and F. Brochard-Wyart, in P.-G. de Gennes Impact on Science, Vol. II, (World Scientific, Singapore, 2009).

[9] T. Sakaue, É. Raphaël, P.-G. de Gennes, and F. BrochardWyart, Europhys. Lett. 72, 83 (2005).

[10] C. Gay, P.-G. de Gennes, É. Raphaël, and F. BrochardWyart, Macromolecules 29, 8379 (1996); P.-G. De Gennes, Adv. Polym. Sci. 138, 91 (1999).

[11] A. P. Markesteijn, O. B. Usta, I. Ali, A. C. Balazs, and J. M. Yeomans, Soft Matter 5, 4575 (2009).

[12] T. D. Long and J. L. Anderson, J. Polym. Sci. B 23, 1261 (1984).

[13] F. Jin and C. Wu, Phys. Rev. Lett. 96, 237801 (2006).

[14] L. Béguin, B. Grassl, F. Brochard-Wyart, M. Rakib, and H. Duval, Soft Matter 7, 96 (2010).

[15] D. R. Latulippe, K. Ager, and A. L. Zydney, J. Membr. Sci. 294, 169 (2007).
[16] J. J. Kasianowicz, J. W. F. Robertson, E. R. Chan, J. E. Reiner, and V. M. Stanford, Annu. Rev. Anal. Chem. 1 737 (2008).

[17] B. Lu, D. P. Hoogerheide, Q. Zhao, H. Zhang, Z. Tang, D. Yu, and J. A. Golovchenko, Nano Lett. 13, 3048 (2013).

[18] M. Firnkes, D. Pedone, J. Knezevic, M. Doblinger, and U. Rant, Nano Lett. 102162 (2010).

[19] V. V. Thacker, S. Ghosal, S. Hernandez-Ainsa, N. A. W. Bell, and U.F. Keyser, Appl. Phys. Lett. 101, 223704 (2012).

[20] G. Ando, C. Hyun, J. Li, and T. Mitsui, ACS Nano 6, 10090 (2012).

[21] V. Kurz, E. M. Nelson, J. Shim, and G. Timp, ACS Nano 7 4057 (2013).

[22] M. J. Levene, J. Korlach, S. W. Turner, M. Foquet, H. G. Craighead, and W. W. Webb, Science 299, 682 (2003).

[23] H. A. Kramers, Physica (Amsterdam) 7, 284 (1940).

[24] P. Rowghanian and A. Y. Grosberg, Phys. Rev. E 87, 042722 (2013).

[25] T. Jovanovic-Talisman, J. Tetenbaum-Novatt, A. S. McKenney, A. Zilman, R. Peters, M. P. Rout, and B. T. Chait, Nature (London) 457, 1023 (2009). 